\title{
Inhibition of Inducible Nitric Oxide Synthase Promotes Vein Graft Neoadventitial Inflammation and Remodelling
}

\author{
Junxi Wu ${ }^{a}$ Roger M. Wadsworth ${ }^{a}$ Simon Kennedy ${ }^{b}$ \\ ${ }^{a}$ Strathclyde Institute of Pharmacy and Biomedical Sciences, University of Strathclyde, and ${ }^{b}$ Department of \\ Integrative and Systems Biology, Faculty of Biomedical and Life Sciences, University of Glasgow, Glasgow, UK
}

\section{Key Words}

Vein graft $\cdot$ Neointima $\cdot$ Adventitia $\cdot$ Endothelium • Inducible nitric oxide synthase $\cdot$ Nitric oxide $\cdot$ Vascular function

\begin{abstract}
Background: Grafting veins into the arterial circulation causes endothelial damage and neointimal hyperplasia. However, the remodelling of vein grafts and the contribution of the endothelium is not well understood. Since nitric oxide (NO) has a crucial role in vascular function, we investigated the importance of NO synthases (NOSs) in vein graft re-endothelialization and remodelling in this study. Methods and Results: Mouse isogenic vena cava was grafted into the carotid artery. Progressive remodelling of the grafted veins was evidenced by re-endothelialization at 2 weeks and subsequent appearance of vasomotor function at 4 weeks. Pharmacological inhibition of inducible NOS (iNOS) with the specific inhibitor 1400W, administered between 2 and 4 weeks after grafting, when re-endothelialization was complete, resulted in neoadventitial inflammation, neoadventitial thickening and impaired functional remodelling. Conclusion: Completion of re-endothelialization is pivotal in vein graft remodelling in the mouse and is associated with a series of changes in inflammation, proliferation and initiation of vascular functional remodelling. After re-endothelial-
\end{abstract}

ization, iNOS upregulation may be an important mechanism to prevent secondary neoadventitial inflammation and preserve ongoing functional remodelling. iNOS activity could therefore be beneficial for long-term patency of the vein graft.

Copyright $\odot 2010$ S. Karger AG, Basel

\section{Introduction}

Vein graft bypass surgery is widely used to treat ischaemic coronary and peripheral artery disease. Although the acute survival rate of vein grafts is high, the long-term patency is not satisfactory, especially for peripheral vein grafts where a high incidence of vein graft disease is present and $30 \%$ of infra-inguinal grafts fail in 2 years [1]. Endothelial denudation is regarded as an important initial injury that induces acute inflammation and allows neointima hyperplasia to proceed $[2,3]$. Neointimal hyperplasia is generally considered as the leading pathology resulting in vein graft diseases such as restenosis and accelerated atherosclerosis.

As regeneration of new endothelium is critical in controlling neointima hyperplasia and vein graft patency [4], the role of nitric oxide (NO) and NO synthases (NOSs) in re-endothelialization and neointima growth are being investigated. Local application of a NO donor that deliv- 
ered NO up to $6 \mathrm{~h}$ after surgery reduced neointimal thickness at 4 weeks in rabbits [5] while incubating the graft for $15 \mathrm{~min}$ in L-arginine (the NOS substrate) polymer solution before implantation suppressed neointima growth [6]. Transfection of grafts with genes for NOSs also suppressed neointima growth up to 4 weeks after surgery in vein grafts and arterial injury models $[7,8]$, even though the transfected gene expression usually lasted for less than 2 weeks [9]. These previous reports seem to suggest that augmenting $\mathrm{NO}$ generation early after grafting has a positive effect on neointima formation.

Conversely, the effects of long-term NO-based treatments on neointima hyperplasia are much less consistent. Four weeks of oral supplementation with L-arginine after surgery caused decreased vascular relaxation to the calcium ionophore A23187, and had no effect on neointima growth [10]. Implantation of an NO-eluting stent in a porcine coronary injury model had no effect on neointima thickness even though a controlled release of NO was present for up to 4 weeks [11]. Since re-endothelialization is usually complete between 2 and 4 weeks in animal models of vein grafting, the inconsistency between shortterm and long-term NO-based treatments raises the question whether NO and NOSs play different roles before and after re-endoethelialization.

Chronic inducible NOS (iNOS) expression in vein graft was observed in both human and animal models $[12,13]$. Induction of iNOS expression in vein grafts was reported to promote re-endothelialization and inhibit neointima hyperplasia [12]. However, the effect of iNOS expression on the sub-acute remodelling after re-endothelialization is largely unexplored. In this study, we aimed to investigate the role of iNOS in vein graft remodelling in the mouse after re-endothelialization. Our findings indicate that iNOS expression after re-endothelialization may regulate the functional remodelling of vein graft wall and the long-term patency, rather than directly affecting the neointimal thickness. This study clarified for the first time the progress of subacute vein graft remodelling and the critical role of iNOS in regulating this process.

\section{Method}

\section{Animals}

Male C57BL/6J (22-28 g) mice were used in this study. All procedures on animals were performed in accordance with the United Kingdom Home Office Guide on the Operation of Animals (Scientific Procedures) Act 1986. Animals were allowed free access to food and water and maintained on a 12-hour light and dark cycle.
Surgery and 1400W Treatment

The surgical procedure was as previously described [2]. Briefly, the thoracic inferior vena cava was harvested from an isogenic donor mouse. The right common carotid artery of the recipient mouse was isolated and cut in the middle. A cuff made from nylon tube was sleeved on the distal arterial end. The artery was reverted back over the cuff and ligated onto the cuff with $8 / 0$ suture. The proximal arterial end was prepared in the same way. The vena cava segment was then sleeved on the arterial ends and fixed onto the cuff with $8 / 0$ suture ligation.

Four consecutive time points at 1-3, 7, 14 and 28 days after vein graft surgery were adopted to characterize the progress of re-endothelialization and remodelling in vein grafts. Six to eight grafted mice at each time point were sacrificed for morphology and immunohistochemistry. For the vascular functional study, 4 mice at 14 days and 8 mice at 28 days were used to test vein grafts for contractile function and another 8 mice at 28 days were used to investigate the relaxation properties of vein grafts.

$\mathrm{N}$-(3-(aminomethyl)benzyl)acetamidine (1400W) was adopted for pharmacological inhibition of iNOS activity in this study. $1400 \mathrm{~W}$ is 1,000 -fold more potent against iNOS than enzyme NOS (eNOS) [14]. For in vivo inhibition of iNOS activity, 1400W has been used at a range of doses from 0.1 to $20 \mathrm{mg} / \mathrm{kg} /$ day [15-17]. The $\mathrm{ED}_{50}$ value of $1400 \mathrm{~W}$ in lipopolysaccharide-induced vascular leakage is about $0.3 \mathrm{mg} / \mathrm{kg}$ [14]. The toxicity of $1400 \mathrm{~W}$ in rodents is minimal. Rats tolerated a dose of $120 \mathrm{mg} /$ day (intravenous infusion) for a 1-week period, and a single dose of $25 \mathrm{mg} / \mathrm{kg} 1400 \mathrm{~W}$ (intravenous injection) had no obvious effects on haemodynamics in rodents [14].

Fourteen days after grafting, one group of 12 mice were administered 1400W (10 mg/kg/day) by subcutaneous injection up to 28 days. Another group of 12 mice were injected with the same volume of physiological saline $(0.9 \% \mathrm{NaCl})$ as control. A third group of mice only had vein graft surgery and served as the nontreated control. Four weeks after surgery, 8 animals from each group were sacrificed for the vascular function study and the remaining 4 were used for immunohistochemistry.

Vascular Functional Study

Vein grafts, thoracic inferior vena cava and carotid arteries were removed and placed in Krebs' solution of the following composition (mM): $\mathrm{NaCl} 118.3, \mathrm{NaHCO}_{3} 25, \mathrm{KCl} 4.7, \mathrm{CaCl}_{2} 2.5, \mathrm{MgSO}_{4}$ $1.2, \mathrm{KH}_{2} \mathrm{PO}_{4} 1.2$ and glucose 11.1. Vessels were cut into $2-\mathrm{mm}$ segments and mounted in a wire-myograph, aerated with $95 \% \mathrm{O}_{2}$ and $5 \% \mathrm{CO}_{2}$ at $37^{\circ} \mathrm{C}$. After normalization, the vessels were set at a level of tension equivalent to $80 \mathrm{~mm} \mathrm{Hg}$ [18]. This step is critical for vein grafts to generate stable contractions and relaxations comparable to the physiological situation. As the luminal diameter and wall thickness of vein grafts varies, the actual circumferential tensions on individual vein graft rings are different for comparable intra-luminal pressures. Contractile function was tested using 3 constrictors added in the following sequence: thromboxane mimetic U46619 (9,11-dideoxy-11 $\alpha$, 9 $\alpha$-epoxymethanoprostaglandin $\left.\mathrm{F}_{2 \alpha}, 3 \times 10^{-7} \mathrm{M}\right)$, phenylephrine $(1 \times$ $\left.10^{-6} \mathrm{M}\right)$, and $\mathrm{KCl}(60 \mathrm{mM})$. In all cases, the vessels were washed at least 3 times to return them to baseline tension prior to addition of the next constrictor. The constrictors were used at high concentrations to test the maximum contractions in vessel rings.

To study vascular relaxant properties, preparations were precontracted to U46619 $\left(3 \times 10^{-8} \mathrm{M}\right)$, which induces a submaximal 
Fig. 1. Dynamics of re-endothelialization and vascular remodelling in vein grafts up to 4 weeks. a HE staining. Acute inflammation at day 7 showing copious infiltrated leukocyte (indicated by arrow). Cells accumulated on both intimal and adventitial sides to form the neointima and neoadventitia. At 14 days a complete layer of endothelial-like cells was present (indicated by arrows). The open arrowheads in a indicate the interface between neointima and neoadventitia. b eNOS staining in contralateral carotid artery and vein grafts at 14 and 28 days. Carotid arteries and vein grafts at 28 days only showed specific eNOS expression localized in the endothelium as indicated by arrows. c Planimetric analysis of graft neointimal and neoadventitial areas. d Intensity of eNOS staining in the re-endothelialized graft at 28 days versus carotid artery. ${ }^{* *} \mathrm{p}<0.01$; ${ }^{+} \mathrm{p}<0.01$, compared with days $1-3$ in $\mathbf{c}$ and compared with carotid artery in $\mathbf{d}(\mathrm{n}=$ 5-8).
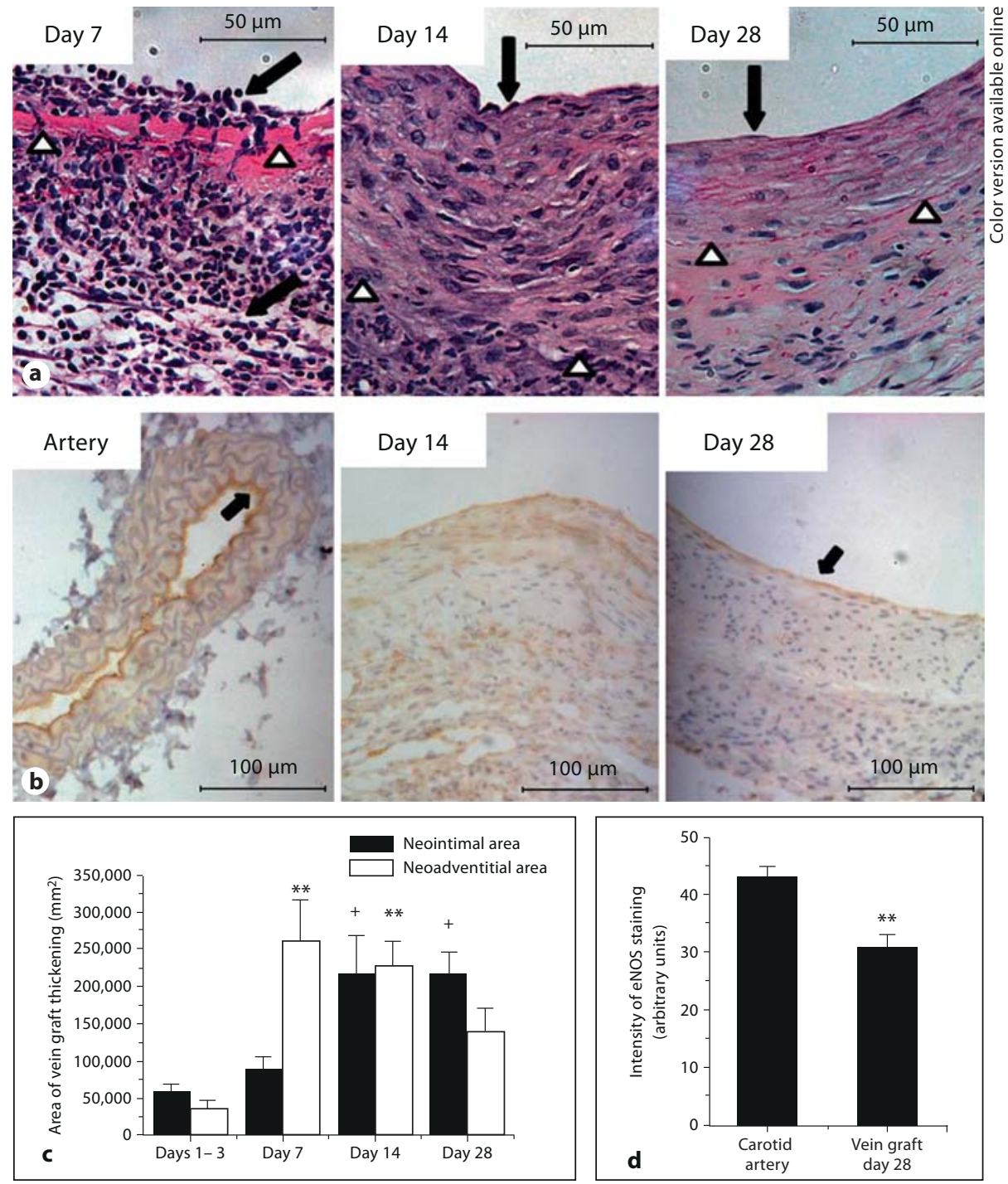

contraction suitable for vessel relaxation experiments. Maximal endothelium-dependent relaxation was then induced by carbachol $\left(1 \times 10^{-5} \mathrm{M}\right)$, and endothelial-independent relaxation by spermine-NONOate (SPNO, an NO donor, $\left.1 \times 10^{-5} \mathrm{M}\right)$. The procedure was repeated after a 30 -min incubation with the NOS inhibitor L-NAME $\left(1 \times 10^{-4} \mathrm{M}\right)$ or with the iNOS inhibitor $1400 \mathrm{~W}$ $\left(1 \times 10^{-5} \mathrm{M}\right)$.

\section{Histology and Immunohistochemistry}

Vein grafts and carotid arteries were fixed overnight in formalin, embedded in paraffin and cut into $4-\mu \mathrm{m}$ sections. Haematoxylin and eosin (HE) staining was applied for morphology and planimetry studies. The area of neointima and neoadventitia were determined using a computerized image-analysis system (Image Pro Plus). The neointima and neoadventitia are defined as the newly formed cell layers on the luminal side and the adventitial side, respectively, of the original vein remnant. As the original vein is only formed by 2-3 layers of cells, no obvious media layer can be defined in this model. In the first week, the original vein remnant appears as a red line with HE staining, which indicates the interface of the neointima and neoadventitia (fig. 1a, day 7). At 2-4 weeks, the original vein is not easily identified, while the neointima and neoadventitia are easily split along the original vein and represent different morphological appearance. The neoadventitia is usually a solid tissue layer with much higher cell density than normal connective tissue. In well-adapted vein grafts, there might be healthy connective tissue surrounding the neoadventitia with a clear interface. In some cases, neoadventitia was found to expand to adjacent muscles, having no connective tissue in between.

For immunohistochemistry, slides were de-waxed, pressure cooked for antigen retrieval, then treated with the appropriate primary antibody followed by a complementary secondary antibody and the peroxidase/DAB chromophore. The antibodies used were: eNOS (610298; BD Transduction Laboratories; 1:100 dilution), iNOS (ab15323; Abcam; 1:50 dilution), von Willebrand fac- 
tor (vWF, A0082; Dako; 1:2,000 dilution), Ki67 (ab15580; Abcam; 1:100 dilution), CD14 (ab25092; Abcam; 1:4,000). The expression level of proteins was quantified using Image-pro Plus by colour matching a stained region, then creating an image mask followed by determination of the total stained area and of the average absorbed pixel intensity [19]. The number of positively stained cells per view area was counted using the same system.

\section{Statistics}

All results are shown as mean \pm standard error of mean and $\mathrm{n}$ indicates the number of animals. Vascular contraction was normalized to the force generated by a $2-\mathrm{mm}$ vessel ring. Vascular relaxation was expressed as a percentage of the U46619-induced contraction. Statistical analysis was performed by one-way ANOVA for multi-group comparison, followed by Tukey's HSD test. Student's t test was applied for 2-group comparisons and the $\chi^{2}$ test was used for detecting differences between proportions. In all cases a p value less than 0.05 was taken to be indicative of statistical significance.

\section{Results}

\section{Re-Endothelialization of the Vein Graft}

The endothelium of the grafted venous segment was denuded at 1-3 days after surgery with acute inflammation and massive infiltration of leukocytes. Cells accumulated on both the luminal side and the adventitial side of the grafted vein, forming a neointima and a neoadventitia. The neointima thickening developed in the first 2 weeks, and stopped growing thereafter (fig. 1a, c). Scattered endothelial cells were present on the lining of the lumen during the first week after surgery, and at 2 weeks there was a complete endothelial layer. Little further leukocyte adhesion at the vein graft lumen was detected from 2 weeks onwards. We defined the 14-day time point as the morphological completion of re-endothelialization. In contrast, accumulation of neoadventitia occurred over a different time scale, reaching its peak at 1 week and gradually decreasing in the following weeks (fig. 1).

The regenerated endothelium at 2 weeks was immature in that specific eNOS was not identified. At 4 weeks, the endothelial layer did stain for eNOS, but the expression level was significantly lower than that in the contralateral carotid artery (fig. 1d).

Consistent with neointimal growth, there was marked cell proliferation in the neointima during the first 2 weeks, but only scattered proliferating cells were found after re-endothelialization. A low level of cell proliferation remained evident at 4 weeks indicating that the vein grafts were not yet stable at this stage (fig. 2a). The neoadventitia developed an extensive network of vasa vasorum, stained by anti-vWF antibody. The number of vasa vasorum decreased significantly after re-endothelialization (fig. 2b).

iNOS expression was induced in the grafted vein at a high though rather variable level during the first week after surgery. This was much reduced after re-endothelialization but with a low level still detectable up to 4 weeks (fig. 2d). Since iNOS is likely to have been induced by proinflammatory mediators, the reduction of iNOS expression after re-endothelialization indicates resolution of acute inflammation, while the weak iNOS expression at 4 weeks suggests that vein grafts may remain under a low level of chronic inflammation. CD14 was adopted as a marker for infiltrated monocytes in the vein graft wall. The very strong and extensive CD14 staining in the first 3 days marked the acute inflammation and leukocyte infiltration early after vein grafting surgery. Up to 2 weeks, the majority of neointimal and neoadventitial cells were still expressing CD14. However, at 4 weeks, the CD14 staining was barely detectable, indicating that these cells had changed their phenotype after re-endothelialization (fig. 2c).

Fully re-endothelialized vein grafts still had impaired vascular function in the 3rd and 4th weeks after grafting. No vein grafts were able to contract to U46619 $(3 \times$ $\left.10^{-8} \mathrm{M}\right), \mathrm{KCl}(60 \mathrm{mM})$ or phenylephrine $\left(1 \times 10^{-6} \mathrm{M}\right)$ at 2 weeks. At 4 weeks, $82.4 \%$ of grafts were able to respond to U46619, but only $14 \%$ of all grafts responded to phenylephrine. As shown in figure 3a, the force generated by vein grafts was in between healthy arteries and vena cava, demonstrating a positive functional remodelling of the vein graft wall. A small endothelium-dependent relaxation to carbachol was observed in vein grafts which was completely abolished by the NOS inhibitor L-NAME (fig. 3b). The relaxation to exogenous NO (SPNO, $1 \times$ $10^{-5} \mathrm{M}$ ) in grafts was not significantly different to that in healthy carotid artery (fig. 3c).

iNOS Inhibition by $1400 \mathrm{~W}$ after Re-Endothelialization $1400 \mathrm{~W}$ treatment had no effect on neointima area, luminal area, neointima morphology or body weight (data not shown). However, the neoadventitial area in $1400 \mathrm{~W}$ treated vein grafts was significantly larger than that seen in control groups (fig. 4a, b).

The intensity of eNOS staining in the endothelium of grafts was unchanged by $1400 \mathrm{~W}$. Cell proliferation, in terms of Ki67-positive cell density, was similar in the neointima of all groups, although highly proliferative cell colonies were present in the neoadventitia in $1400 \mathrm{~W}$-treated vein grafts (fig. 4a). Interestingly, the number of vasa vasorum stained by anti-vWF antibody in the neoadventitia 


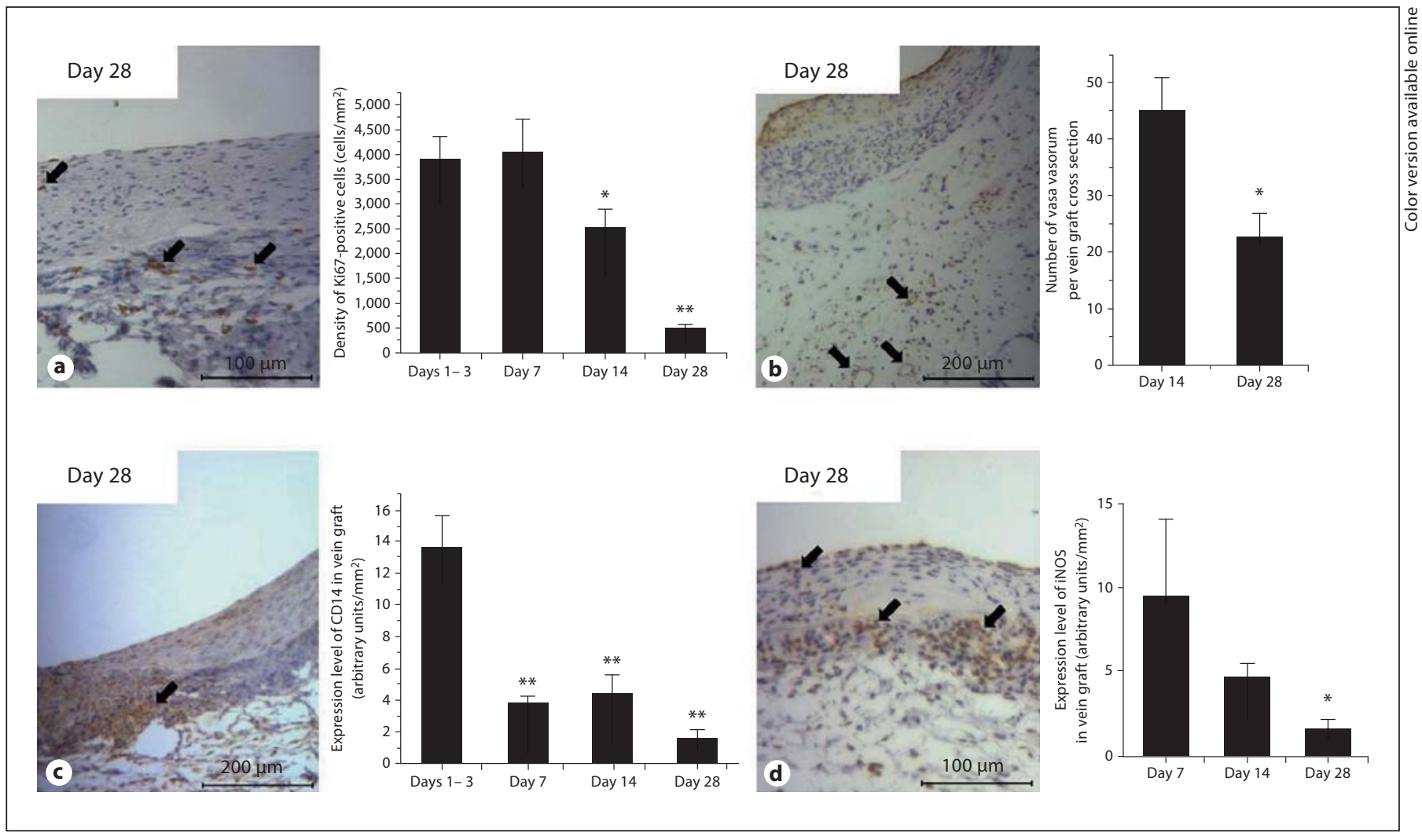

Fig. 2. Representative immunohistochemistry staining and quantitative analysis of Ki67 (a), vWF (b), CD14 (c) and iNOS (d) expression. Arrows indicate positive staining indicated by the DAB chromogen. ${ }^{*} \mathrm{p}<0.05,{ }^{* *} \mathrm{p}<0.01$, compared with days $1-3$ in a and $\mathbf{c}$, compared with day 14 in $\mathbf{b}$, and compared with day 7 in $\mathbf{d}$ $(\mathrm{n}=4-8)$.



Fig. 3. Functional responses in carotid arteries and vein grafts. a U46619 $\left(3 \times 10^{-7} \mathrm{M}\right)$ induced contraction in carotid artery, 4 -week-old vein graft and vena cava. ${ }^{* *} \mathrm{p}<0.01$, compared with carotid artery; ${ }^{+} \mathrm{p}<0.05$, compared with vein graft. b Relaxation induced by carbachol $\left(1 \times 10^{-5} \mathrm{M}\right)$, with or without the presence of L-NAME $\left(1 \times 10^{-4} \mathrm{M}\right){ }^{* *} \mathrm{p}<0.01$, compared with carbacholinduced relaxation in carotid artery; ${ }^{\circ} \mathrm{p}<0.05$, compared with carbachol-induced relaxation in vein graft. $\mathbf{c}$ Relaxation induced by SPNO (NO donor, $\left.1 \times 10^{-5} \mathrm{M}\right)$, with or without the presence of L-NAME $\left(1 \times 10^{-4} \mathrm{M}\right)(\mathrm{n}=6)$. 
Fig. 4. The effects of iNOS inhibition after re-endothelialization. a Representative photomicrographs of the $1400 \mathrm{~W}$-treated vein grafts stained for $\mathrm{HE}, \mathrm{Ki} 67, \mathrm{vWF}$, CD14 and iNOS. The arrowheads indicate the border of the neointima, while the arrows indicate positive staining. 1400W treatment significantly increased neoadventitial area (b) and the number of vasa vasorum (c). The expression of iNOS was up-regulated in both saline-treated and $1400 \mathrm{~W}$-treated vein grafts $(\mathbf{d}) .{ }^{*} \mathrm{p}<0.05$, ${ }^{* *} \mathrm{p}<0.01$, compared with VG-non; ${ }^{+} \mathrm{p}<$ 0.05 , compared with VG-saline $(\mathrm{n}=4-7)$.

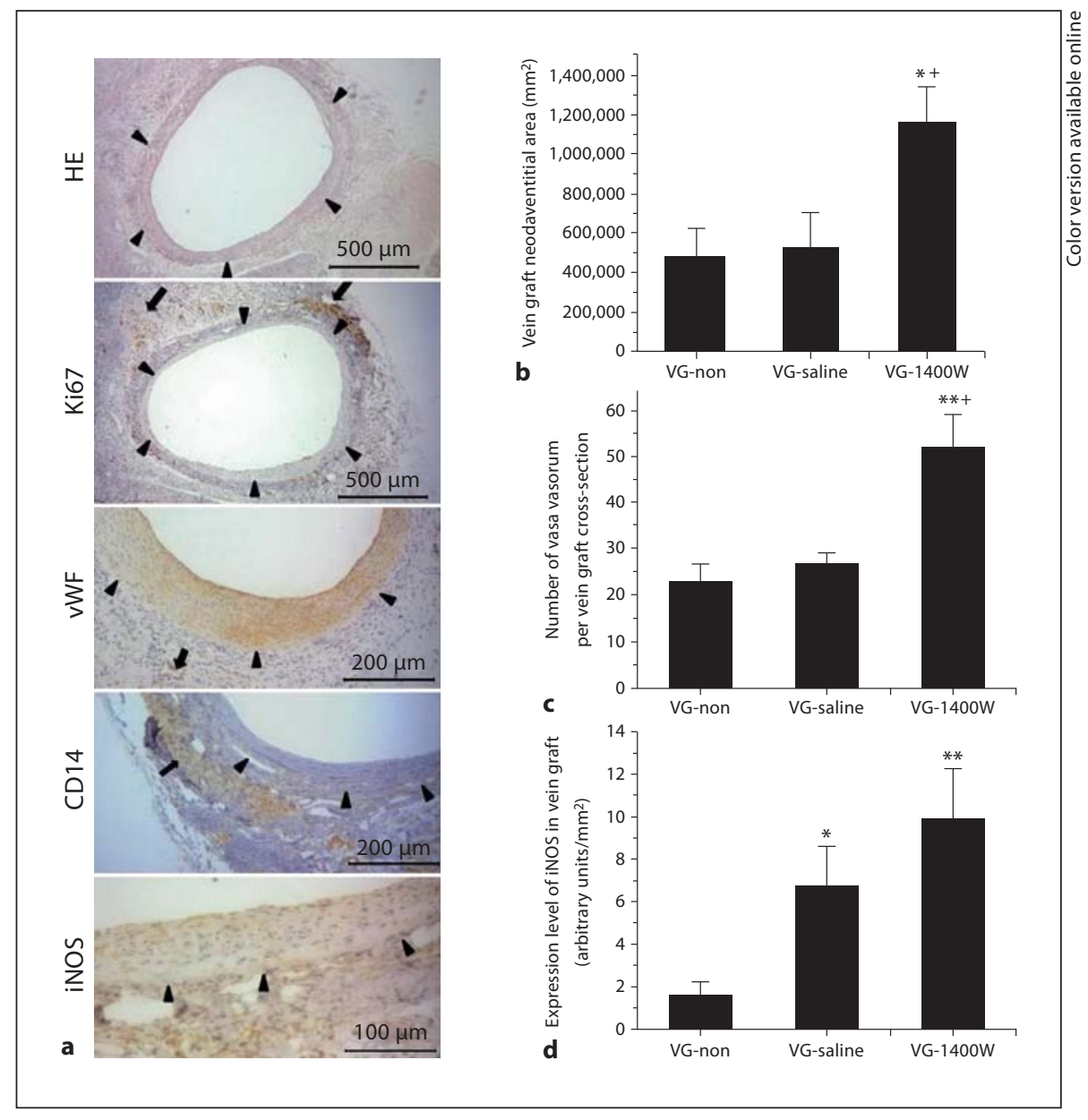

of $1400 \mathrm{~W}$-treated vein grafts was significantly higher compared to non-treated and saline-treated vein grafts (fig. 4a, c). iNOS expression was significantly increased in both saline-treated and $1400 \mathrm{~W}$-treated vein grafts, compared with non-treated vein grafts (fig. $4 \mathrm{a}, \mathrm{d}$ ). Although there was no difference in CD14 expression over the whole vein graft wall, multiple cell colonies in the neoadventitia in $1400 \mathrm{~W}$-treated vein grafts exhibited intensive CD14 staining, indicating newly infiltrated leukocytes and local inflammation in the neoadventitia (fig. 4a).

One hundred percent of non-treated vein grafts and $63 \%$ of saline-treated vein grafts gave substantial contraction $(\geq 0.5 \mathrm{mN})$ to $\mathrm{U} 46619\left(3 \times 10^{-8} \mathrm{M}\right)$ as shown in table 1 , while only $43 \% 1400 \mathrm{~W}$-treated vein grafts contracted strongly to U46619 ( $\mathrm{p}<0.05$ vs. non-treated group). The vein grafts that contracted less than $0.5 \mathrm{mN}$ usually could not maintain the contraction, and thus it was not possible to measure their relaxation. In 1400Wtreated vein grafts that were substantially contracted by
Table 1. The percentage of 4 -week-old vein grafts that showed substantial contraction $(\geq 0.5 \mathrm{mN})$ to $\mathrm{U} 46619\left(3 \times 10^{-8} \mathrm{M}\right)$

$$
\begin{array}{lll}
\begin{array}{l}
\text { Non-treated } \\
(\mathrm{n}=8)
\end{array} & \begin{array}{l}
\text { Saline-treated } \\
(\mathrm{n}=8)
\end{array} & \begin{array}{l}
\text { 1400W-treated } \\
(\mathrm{n}=7)
\end{array}
\end{array}
$$

Percentage of vein grafts

that contract $\geq 0.5 \mathrm{mN} \quad 100 \% \quad 63 \% \quad 43 \%$ *

${ }^{*} \mathrm{p}<0.05$, compared with non-treated vein graft.

$\mathrm{U} 446619\left(3 \times 10^{-8} \mathrm{M}\right)$, no difference in the force of contraction was observed compared with other groups. Endothelium-dependent relaxation induced by carbachol was observed in all vein grafts and this was abolished by L-NAME incubation. There was no significant difference in either the carbachol-induced relaxation or SPNO-induced relaxation in any of the 3 groups (fig. 5). 


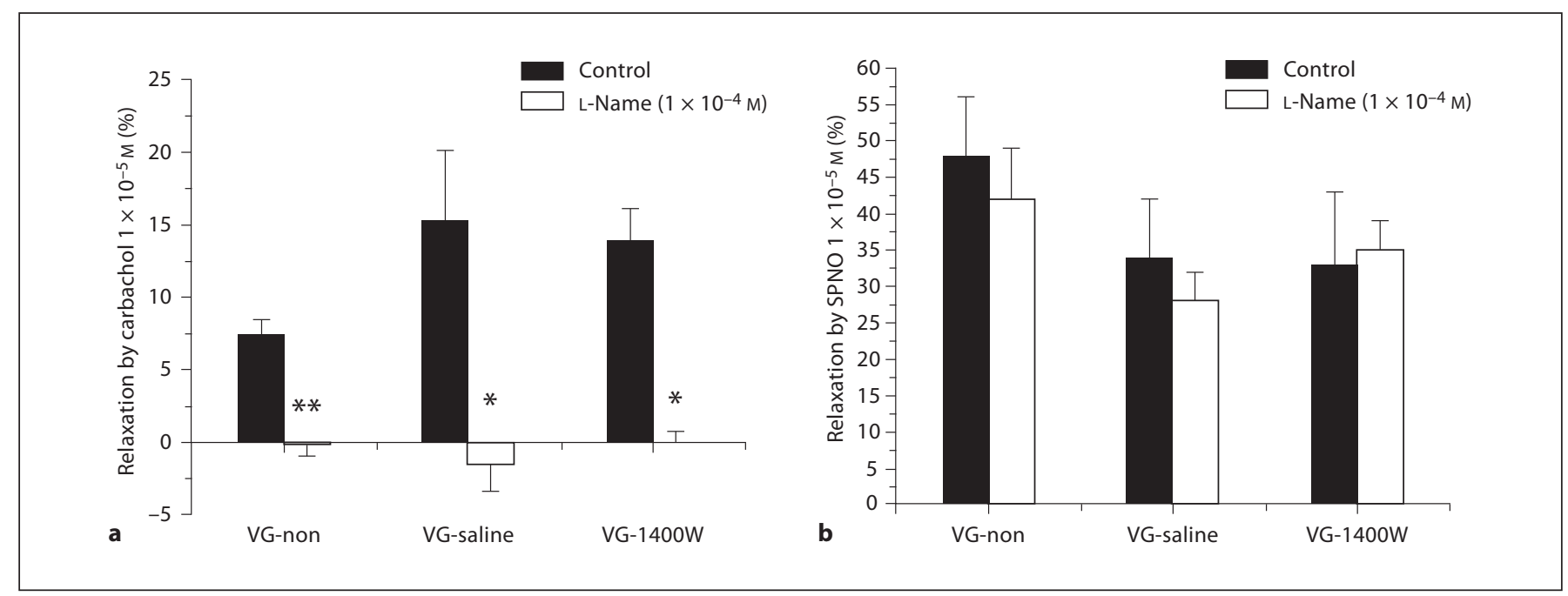

Fig. 5. Relaxation induced by carbachol (a) or SPNO (b) with or without the presence of L-NAME $\left(1 \times 10^{-4} \mathrm{M}\right)$. In vessels from control and $1400 \mathrm{~W}$-treated animals. ${ }^{*} \mathrm{p}<0.05,{ }^{* *} \mathrm{p}<0.01$, compared with control (VG-non, $\mathrm{n}=6$; VG-saline, $\mathrm{n}=5$; VG-1400W, $\mathrm{n}=3$ ).

\section{Discussion}

\section{Re-Endothelialization and Neointima Hyperplasia}

This study is the first to report that re-endothelialization in the mouse was associated with a series of changes in vein graft morphology, protein expression and vascular function. Endothelial-like cells were first observed on the luminal surface in some vein grafts 1 week after surgery with re-endothelialization complete in all vein grafts at 2 weeks, similar to previous studies [20,21]. As accelerated re-endothelialization was shown to be associated with reduced neointima formation [22], our study strengthens the dynamic relationship between re-endothelialization and neointimal hyperplasia. Completion of re-endothelialization appears to halt neointimal hyperplasia and is associated with a series of changes in tissue morphology, protein expression, cell activity and vascular function. Adherent leukocytes were not present in reendothelialized vein grafts observed at 2 weeks (fig. 1a) and, coupled with the significant reduction in CD14 and iNOS expression, these observations were clear signs of the resolution of acute inflammation. The reduction of cell proliferation and adventitial vasa vasorum density may also directly contribute to the prevention of neointimal accumulation after re-endothelialization. However, chronic inflammation was still present with low levels of iNOS expression and cell proliferation in the vein graft wall up to 4 weeks. Since eNOS was not observed in the regenerated endothelium until 4 weeks, eNOS may not be crucial in regulating the re-endothelialization or the remodelling of the underlying layers of the vein graft.

\section{Vascular Functional Remodelling after \\ Re-Endothelialization}

Another important finding in this study is the emergence of vascular function in the graft after re-endothelialization. At 2 weeks, no vein graft showed any vasomotor function. However, at 4 weeks, over $80 \%$ of all vein grafts were able to give an obvious contraction $(>0.5 \mathrm{mN})$ in response to U46619 $\left(3 \times 10^{-8} \mathrm{M}\right)$, and $14 \%$ of all tested vein graft rings were also able to respond to phenylephrine $\left(1 \times 10^{-6} \mathrm{M}\right)$. However, no vein graft contracted to $\mathrm{KCl}(60 \mathrm{mM})$ up to 4 weeks. These data suggest that initiation of vascular functional remodelling in vein grafts occurred subsequent to re-endothelialization. The different responses to the 3 vasoconstrictors might indicate different remodelling speed in various signalling pathways. The thromboxane receptor-mediated contraction (by $\mathrm{U} 46619$, a thromboxane $\mathrm{A}_{2}$ mimetic) is most probably the first functional response to appear and may indicate the differentiation of neointimal cells into smooth muscle cells. Recently, Kim et al. [23] reported that thromboxane $\mathrm{A}_{2}$ induces differentiation of stem cells to smooth muscle cells. Since at least $20 \%$ of the neointimal cells originate from bone marrow-derived cells, it would be very interesting to investigate if the thromboxane signalling pathway is involved in cell differentiation and functional remodelling in vein grafts. The appearance of specific 
eNOS expression in the new endothelium was accompanied by the emergence of endothelial-dependent and NOmediated vascular relaxation. However, the carbachol $\left(1 \times 10^{-5} \mathrm{M}\right)$-induced endothelium-dependent relaxation was only about $5 \%$ of the precontraction. Since the relaxation to exogenous NO (by spermine-NONOate) was largely preserved, the poor endothelium-dependent relaxation is not due to the disruption of the NO signalling pathway in smooth muscle cells.

\section{The Regulative Role of $i N O S$ after}

\section{Re-Endothelialization}

Sustained iNOS expression was reported in both human and animal vein grafts $[12,13]$. Abrogation of iNOS activity in the early weeks, either by pharmacological inhibition or genetic deletion of iNOS gene, exacerbated neointima hyperplasia $[12,15]$.

Our data confirmed that iNOS expression persisted after completion of re-endothelialization in the vein graft wall. As the long-term effect of NO is controversial, pharmacological inhibition of iNOS function was introduced after re-endothelialization (in the 3rd and 4th post-operative weeks). Using this protocol, the neointima formation prior to re-endothelialization was not affected, a period during which iNOS is known to have an inhibitory effect. $1400 \mathrm{~W}$ was previously reported to inhibit the function of iNOS rather than affecting the protein expression [24], and our data confirmed that the expression level of iNOS was not affected by $1400 \mathrm{~W}$ treatment.

Interestingly, iNOS expression was significantly upregulated in the saline control group compared to the non-treated group. No changes were observed in other parameters, such as vascular function, eNOS expression, cell proliferation and neointima and neoadventitial area. It is well known that expression of iNOS is usually induced by pro-inflammatory cytokines [25] and neointima cells are reported to be more sensitive to pro-inflammatory stimuli in iNOS induction, compared with normal smooth muscle cells [26]. The up-regulation of iNOS in saline-treated vein grafts was most probably due to stimulation caused by daily subcutaneous injection, which might introduce a low-grade secondary inflammation. Although the $1400 \mathrm{~W}$-treated group received the same manipulation/stimulation, inhibition of iNOS function resulted in 2 additional consequences compared with the saline-treated group: (1) impaired vascular functional remodelling and (2) neoadventitial inflammation as well as hyperplasia. These data indicate that the vein graft wall is vulnerable to pro-inflammatory stimuli even after re-endothelialization, and iNOS induction is an im- portant mechanism to protect vein grafts from unfavourable remodelling caused by secondary inflammation.

In the present study, the neoadventitia seems more vulnerable to inflammation than the neointima. In human vein grafts, the adventitial vasa vasorum was more important than the luminal endothelium for leukocyte recruitment [27]. This may be explained by the different endothelial phenotypes. It is well known that the endothelium of the post-capillary venules rather than large arteries is the classic location for leukocyte adhesion and infiltration. In our experiment, the multiple colonies of CD14-positve cells in the neoadventitia in the 1400Wtreated group also confirmed that leukocyte infiltration did occur in the neoadventitia but not in the neointima. Our results suggest that iNOS could inhibit neoadventitial inflammation, although the detailed mechanism requires further investigation.

An increase in the number of vasa vasorum in the neoadventitia was another consequence of iNOS inhibition. The increased angiogenesis could be secondary to neoadventitial inflammation and hyperplasia, due to the increased metabolic requirements. However, the contribution of vasa vasorum to inflammation and neointimal hyperplasia is likely to be considerable [28]. In humans, the number of vasa vasorum in the adventitia and media of stenotic vein grafts was positively correlated with neointima thickness, while there was no association in nonstenotic vein grafts [29]. Adventitial angiogenesis-dependent neointimal thickening was also observed in animal models [30]. Furthermore, adventitial neovascularization of vasa vasorum was reported to be a part of the early atherosclerotic remodelling process in the presence of hyperlipidemia [31]. NO is known to regulate microvascular permeability and angiogenesis. It was reported that eNOS, but not iNOS, increased microvascular permeability in inflammation [32]. VEGF was reported to increase angiogenesis as well as vascular permeability in both iNOS ${ }^{+/+}$and iNOS ${ }^{-/-}$mice, but not in $\mathrm{eNOS}^{-/-}$mice [33]. This indicates that eNOS and iNOS are playing different roles in regulating microvascular function and angiogenesis.

Collectively, our results demonstrate that the endothelium plays a very important role in vein graft remodelling. Completion of re-endothelialization shifted vein grafts from neointima thickening to vascular functional remodelling. After re-endothelialization, iNOS up-regulation may be an important mechanism to prevent secondary neoadventitial inflammation and preserve ongoing functional remodelling and thus could be beneficial for long-term patency of vein grafts. 


\section{References}

$\checkmark 1$ Mills JL Sr, Wixon CL, James DC, Devine J, Westerband A, Hughes JD: The natural history of intermediate and critical vein graft stenosis: recommendations for continued surveillance or repair. J Vasc Surg 2001;33: 273-278; discussion 278-280.

>2 Zou Y, Dietrich H, Hu Y, Metzler B, Wick G, $\mathrm{Xu}$ Q: Mouse model of venous bypass graft arteriosclerosis. Am J Pathol 1998;153:13011310.

3 Zhang L, Hagen PO, Kisslo J, Peppel K, Freedman NJ: Neointimal hyperplasia rapidly reaches steady state in a novel murine vein graft model. J Vasc Surg 2002;36:824832 .

4 Ohno N, Itoh H, Ikeda T, Ueyama K, Yamahara K, Doi K, Yamashita J, Inoue M, Masatsugu K, Sawada N, Fukunaga Y, Sakaguchi S, Sone M, Yurugi T, Kook H, Komeda M, Nakao K: Accelerated reendothelialization with suppressed thrombogenic property and neointimal hyperplasia of rabbit jugular vein grafts by adenovirus-mediated gene transfer of c-type natriuretic peptide. Circulation 2002;105:1623-1626.

5 Fulton GJ, Davies MG, Barber L, Gray JL, Svendsen E, Hagen PO: Local effects of nitric oxide supplementation and suppression in the development of intimal hyperplasia in experimental vein grafts. Eur J Vasc Endovasc Surg 1998;15:279-289.

6 Kown MH, Yamaguchi A, Jahncke CL, Miniati D, Murata S, Grunenfelder J, Koransky ML, Rothbard JB, Robbins RC: L-arginine polymers inhibit the development of vein graft neointimal hyperplasia. J Thorac Cardiovasc Surg 2001;121:971-980.

7 Wu S, Wang X, Guo L, Zi J: Adenovirus mediated endothelial nitric oxide synthase gene transfer prevents restenosis of vein grafts. Asaio J 2004;50:272-277.

$\checkmark 8$ Barbato JE, Tzeng E: Inos gene transfer for graft disease. Trends Cardiovasc Med 2004; 14:267-272.

9 Channon KM, Fulton GJ, Gray JL, Annex $\mathrm{BH}$, Shetty GA, Blazing MA, Peters KG, Hagen PO, George SE: Efficient adenoviral gene transfer to early venous bypass grafts: comparison with native vessels. Cardiovasc Res 1997;35:505-513.

10 Landis ME, Ray EC, Gloviczki P, Miller VM: Effects of dietary L-arginine on structure and function of flow-restricted vein grafts. J Vasc Surg 2001;33:829-839.
1 Yoon JH, Wu CJ, Homme J, Tuch RJ, Wolff RG, Topol EJ, Lincoff AM: Local delivery of nitric oxide from an eluting stent to inhibit neointimal thickening in a porcine coronary injury model. Yonsei Med J 2002;43:242251.

12 Mayr U, Zou Y, Zhang Z, Dietrich H, Hu Y, $\mathrm{Xu}$ Q: Accelerated arteriosclerosis of vein grafts in inducible no synthase (-/-) mice is related to decreased endothelial progenitor cell repair. Circ Res 2006;98:412-420.

13 Dattilo JB, Dattilo MP, Spratt JA, Matsuura J, Yager DR, Makhoul RG: Inducible nitric oxide synthase expression in human vein grafts. Am J Surg 1997;174:177-180.

14 Garvey EP, Oplinger JA, Furfine ES, Kiff RJ, Laszlo F, Whittle BJ, Knowles RG: 1400W is a slow, tight binding, and highly selective inhibitor of inducible nitric-oxide synthase in vitro and in vivo. J Biol Chem 1997;272: 4959-4963.

15 Moore ZW, Hui DY: Apolipoprotein E inhibition of vascular hyperplasia and neointima formation requires inducible nitric oxide synthase. J Lipid Res 2005;46:2083-2090.

16 Guo JS, Cho CH, Wang JY, Koo MW: Differential effects of selective and non-selective inhibition of nitric oxide synthase on the expression and activity of cyclooxygenase-2 during gastric ulcer healing. Eur J Pharmacol 2006;536:301-308.

-17 Sakaguchi Y, Shirahase H, Ichikawa A, Kanda M, Nozaki Y, Uehara Y: Effects of selective iNOS inhibition on type II collagen-induced arthritis in mice. Life Sci 2004;75:2257-2267.

18 Mulvany MJ, Halpern W: Contractile properties of small arterial resistance vessels in spontaneously hypertensive and normotensive rats. Circ Res 1977;41:19-26.

19 Lehr HA, Mankoff DA, Corwin D, Santeusanio G, Gown AM: Application of Photoshopbased image analysis to quantification of hormone receptor expression in breast cancer. J Histochem Cytochem 1997;45:15591565 .

20 Westerband A, Crouse D, Richter LC, Aguirre ML, Wixon CC, James DC, Mills JL, Hunter GC, Heimark RL: Vein adaptation to arterialization in an experimental model. J Vasc Surg 2001;33:561-569.

21 Ehsan A, Mann MJ, Dell'Acqua G, Tamura K, Braun-Dullaeus R, Dzau VJ: Endothelial healing in vein grafts: proliferative burst unimpaired by genetic therapy of neointimal disease. Circulation 2002;105:1686-1692.

$\checkmark 22$ Hutter R, Carrick FE, Valdiviezo C, Wolinsky C, Rudge JS, Wiegand SJ, Fuster V, Badimon JJ, Sauter BV: Vascular endothelial growth factor regulates reendothelialization and neointima formation in a mouse mode of arterial injury. Circulation 2004;110: $2430-2435$.
23 Kim MR, Jeon ES, Kim YM, Lee JS, Kim JH: Thromboxane $\mathrm{A}_{2}$ induces differentiation of human mesenchymal stem cells to smooth muscle-like cells. Stem Cells 2009;27:191199.

24 Fries DM, Paxinou E, Themistocleous M, Swanberg E, Griendling KK, Salvemini D, Slot JW, Heijnen HF, Hazen SL, Ischiropoulos $\mathrm{H}$ : Expression of inducible nitric-oxide synthase and intracellular protein tyrosine nitration in vascular smooth muscle cells: role of reactive oxygen species. J Biol Chem 2003;278:22901-22907.

25 Kleinert H, Pautz A, Linker K, Schwarz PM: Regulation of the expression of inducible nitric oxide synthase. Eur J Pharmacol 2004; 500:255-266.

26 Yan Z, Hansson GK: Overexpression of inducible nitric oxide synthase by neointimal smooth muscle cells. Circ Res 1998;82:21-29.

27 Chester AH, Morrison KJ, Yacoub MH: Expression of vascular adhesion molecules in saphenous vein coronary bypass grafts. Ann Thorac Surg 1998;65:1685-1689.

28 Fuchs S, Kornowski R, Leon MB, Epstein SE: Anti-angiogenesis: a new potential strategy to inhibit restenosis. Int J Cardiovasc Intervent 2001;4:3-6.

29 Westerband A, Gentile AT, Hunter GC, Gooden MA, Aguirre ML, Berman SS, Mills JL: Intimal growth and neovascularization in human stenotic vein grafts. J Am Coll Surg 2000;191:264-271.

30 Khurana R, Simons M, Martin JF, Zachary IC: Role of angiogenesis in cardiovascular disease: a critical appraisal. Circulation 2005;112:1813-1824.

- 31 Kwon HM, Sangiorgi G, Ritman EL, McKenna C, Holmes DR Jr, Schwartz RS, Lerman A: Enhanced coronary vasa vasorum neovascularization in experimental hypercholesterolemia. J Clin Invest 1998;101: 1551-1556

32 Hatakeyama T, Pappas PJ, Hobson RW 2nd, Boric MP, Sessa WC, Duran WN: Endothelial nitric oxide synthase regulates microvascular hyperpermeability in vivo. J Physiol 2006;574:275-281.

-33 Fukumura D, Gohongi T, Kadambi A, Izumi Y, Ang J, Yun CO, Buerk DG, Huang PL, Jain RK: Predominant role of endothelial nitric oxide synthase in vascular endothelial growth factor-induced angiogenesis and vascular permeability. Proc Natl Acad Sci USA 2001;98:2604-2609. 